\title{
Serum periostin levels in adolescents
}

\author{
Yosuke Nakamura, ${ }^{1}$ Yuko Yokoyama, ${ }^{1}$ Kenichiro Nakajima, ${ }^{1,2}$ Tadao Enomoto, ${ }^{1,3}$ Kazunori Fujiwara, ${ }^{1}$ Hiromi Takeuchi ${ }^{1}$
}

\begin{abstract}
Background: The significance of periostin as a biomarker of Th2-induced airway inflammation has recently been highlighted in adult patients with allergic diseases. It may help identify drug-responsive inflammatory phenotypes, particularly in children. However, little is known about the usefulness of this parameter as a biomarker for allergic diseases in children. Furthermore, it is not known how much adolescent bone metabolism affects allergic inflammation.
\end{abstract}

Objective: To evaluate the relationship between serum periostin levels and allergic diseases in adolescents, we investigated periostin levels and the prevalence of allergic diseases.

Methods: We conducted a cross-sectional observational study of 100 males and females in two age groups: age 9-12 years (pre-early adolescence) and 13-15 years (post-early adolescence). Serum periostin levels were determined using Enzyme-Linked Immuno Sorbent Assay (ELISA). Presence of allergic diseases and allergy sensitization were obtained via a self-reported survey and the Multiple Antigen Simultaneous Test (MAST). The protocol was registered in a clinical trial registry as UMIN 000036051.

Results: There were no significant differences in serum periostin levels between pre-early and post-early adolescents. There were no differences by gender. Age and Body Mass Index were not significantly associated with serum periostin levels. Periostin levels were elevated in adolescents with allergic diseases overall compared to healthy adolescents [mean (95\%CI): $41.6(33.4,49.7)$ vs. $28.6(21.9,35.3) \mathrm{ng} / \mathrm{ml} ; P<0.05]$.

Conclusions: The effects of bone metabolism on serum periostin levels may be limited at 9-15 years of age. Further studies are required to determine reference values in adolescents.

Key words: Adolescent, Allergic rhinitis, Epidemiology, Metabolism, Periostin

\section{From:}

${ }^{1}$ Division of Otolaryngology, Head and Neck Surgery,

Faculty of Medicine, Tottori University,

${ }^{2}$ Division of Otolaryngology, Matsue Red Cross Hospital, Shimane

${ }^{3}$ NPO Japan Health Promotion Supporting Network

\author{
Corresponding author: \\ Yosuke Nakamura \\ Division of Otolaryngology, Head and Neck Surgery, \\ Faculty of Medicine, Tottori University \\ 36-1 Nishicho, Yonago, Tottori 683-8504, Japan \\ E-mail: yosuken@tottori-u.ac.jp
}

\section{Introduction}

Increases in allergic diseases such as atopic dermatitis (AD), bronchial asthma (BA), and allergic rhinitis (AR) over the last 30-40 years have been well documented worldwide. ${ }^{1}$ Periostin is a matricellular protein that plays an important role in the development of allergic diseases. ${ }^{2}$ Recent data have shown that periostin is a serum biomarker of remodeling in various allergic diseases, such as $\mathrm{BA}, \mathrm{AR}, \mathrm{AD}$ and chronic sinusitis in adults. ${ }^{3}$ In studies based on the International Study of Asthma and Allergies in Childhood (ISAAC), the relationship between serum periostin levels and allergic diseases has not been clarified. ${ }^{1,4-6}$ However, a few investigations have suggested that periostin is associated with the severity of allergic diseases and is a diagnostic biomarker for BA and $\mathrm{AD}$ in children..$^{7-10}$ Little consideration has been given to adolescents, and a reference range for periostin has not been established for adolescents. ${ }^{3}$ We investigated the relationship between periostin levels and allergic diseases in early adolescents.

\section{Methods}

We conducted a single-center, cross-sectional, observational study. 


\section{Study population}

We recruited 100 participants for a health survey on child growth. We studied 50 males and 50 females in two age groups: age 9-12 years (pre-early adolescence) and 13-15 years (post-early adolescence). Participants were in fourth grade or above. We excluded participants with infectious diseases. A study questionnaire was distributed and completed by parents. Blood collection and physical examinations were performed from January to March 2017. The details of this study were explained to the prospective participants and their parents. Written informed consent was obtained from parents. The study protocol (No. 1603B047) was approved by the Committee on Human Research of Tottori University in accordance with the Declaration of Helsinki. Data collection was performed in accordance with the principle of respect for the confidentiality of patient records.

\section{Assessment}

The diagnosis of allergic disease was made based on the Japanese version of the ISAAC ${ }^{1}$ core written questionnaire and Japanese guidelines for allergic rhinitis ${ }^{11}$ (Figure 1). Lifetime prevalence was estimated by counting the number of participants with physician-diagnosed disease based on questions 1, 4, 7, and 11 of the questionnaire (Figure 1).
The definition of remission was based on the assumption that a physician had diagnosed allergic disease in the past and there were no symptoms for more than one year. The questionnaire also included questions about grade, age, gender, height, and weight.

A peripheral blood sample was collected from all participants at the time of questionnaire administration. Samples were spun at $3000 \mathrm{rpm}$ for 5 minutes with serum, placed in $500 \mu \mathrm{L}$ aliquots, and frozen at $-30^{\circ} \mathrm{C}$ until testing.

Serum periostin was measured using a human ELISA kit (R\&D Systems, Minneapolis, MN, USA). All procedures were performed according to the manufacturer's instructions.

Serum total IgE and specific IgE levels were measured. A total of 36 common allergens were evaluated using Multiple Antigen Simultaneous Test (MAST) Immunosystems IV (Kyowa Medex, Tokyo, Japan): Dermatophagoides farinae, house dust mite, cat, dog, Japanese cedar, cypress, alder, birch, orchard grass, timothy grass, ragweed, mugwort, Alternaria, Aspergillus, Candida, latex, egg white, ovomucoid, milk, wheat, peanut, soya, soba, gum, rice, shrimp, crab, kiwi, banana, peach, tomato, tuna, salmon, beef, chicken meat, and pork. Serum specific IgE levels greater than class 2 were considered positive. Early adolescents without any allergic diseases or allergic sensitization were defined as healthy adolescents.

\begin{tabular}{|c|c|c|}
\hline \multicolumn{3}{|c|}{ 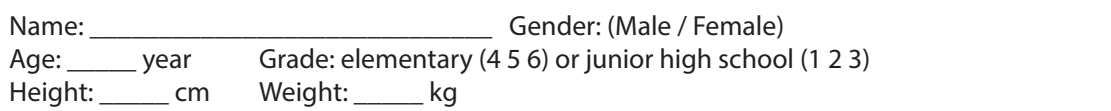 } \\
\hline $\begin{array}{l}\text { Allergic diseases } \\
\text { Q1 Have you ever be } \\
\text { Q2 Have you had wh } \\
\text { IF YOU ANSWERED“N } \\
\text { Q3 What medication }\end{array}$ & $\begin{array}{l}\text { Nen diagnosed with bronchial asthma by a doctor? } \\
\text { neezing or whistling in the chest in the last } 12 \text { months? } \\
\text { so haEAS SKIP TO QUESTION } 4 \text {. } \\
\text { shour child taking? }\end{array}$ & $\begin{array}{l}\text { Yes / No } \\
\text { Yes / No }\end{array}$ \\
\hline $\begin{array}{l}\text { Q4 Have you ever be } \\
\text { Q5 Have you had ato } \\
\text { IF YOU ANSWERED“N } \\
\text { Q6 What medication }\end{array}$ & $\begin{array}{l}\text { Den diagnosed with atopic eczema by a doctor? } \\
\text { opic eczema in the last } 12 \text { months? } \\
\text { NO" PLEASE SKIP TO QUESTION } 7 . \\
\text { s has your child taking? }\end{array}$ & $\begin{array}{l}\text { Yes / No } \\
\text { Yes / No }\end{array}$ \\
\hline $\begin{array}{l}\text { Q7 Have you ever be } \\
\text { Q8 Have you had rhir } \\
\text { IF YOU ANSWERED“N } \\
\text { Q9 Tell me about the } \\
\text { Sneezing: } \\
\text { Nasal bloc } \\
\text { Q10 Please list some }\end{array}$ & $\begin{array}{l}\text { en diagnosed with allergic rhinitis or hay fever by a doctor? } \\
\text { initis symptoms in the last } 12 \text { months? } \\
\text { NO" PLEASE SKIP TO QUESTION } 11 . \\
\text { efrequency of sneezing and runny nose and the degree of na } \\
\text { :( ) times/day, Rhinorrhea: ( ) times/day, } \\
\text { ckage: (none / mild / moderate / severe / most severe) } \\
\text { things that causes the rhinitis: }\end{array}$ & $\begin{array}{l}\text { Yes / No } \\
\text { Yes / No } \\
\text { ongestion. }\end{array}$ \\
\hline $\begin{array}{l}\text { Q11 Have you ever b } \\
\text { Q12 Does the symptc } \\
\text { Q13 Please list the fo }\end{array}$ & $\begin{array}{l}\text { oeen diagnosed with food allergies by a doctor? } \\
\text { oms still occur? } \\
\text { oods that have caused problems for your child: }\end{array}$ & $\begin{array}{l}\text { Yes / No } \\
\text { Yes / No }\end{array}$ \\
\hline
\end{tabular}

Figure 1. Survey questionnaire.

The questionnaire was modified in accordance with the International Study of Asthma and Allergies in Childhood (ISAAC) core written questionnaire and Japanese guidelines for allergic rhinitis. Height and weight were also measured at the same time. 


\section{Statistical analysis}

Demographic data were presented as means \pm SE. Differences in serum periostin levels were compared using Welch's $t$-test. Correlations between serum periostin level and age were analyzed using the Spearman correlation test. Multivariated linear regression analysis was used to show an independent effect after adjusting for confounding variables. Fisher's least significant difference test was used to compare rates of allergen-specific IgE positivity. A two-sided significance level was set at 0.05 . All statistical analyses were performed with IBM SPSS version 25 (IBM Corp. Armonk, NY, USA).

\section{Results}

Of 100 participants in early adolescence included in the study, 50 were male and 50 were female. Demographic data are presented in Table 1.

Table 1. Clinical characteristics.

\begin{tabular}{|c|c|c|c|c|}
\hline & Overall & Males & Females & $P$ value \\
\hline Sample size & 100 & 50 & 50 & 1.00 \\
\hline Age (years) & $12.3 \pm 0.2$ & $12.4 \pm 0.2$ & $12.2 \pm 0.2$ & 0.58 \\
\hline Height $(\mathrm{cm})$ & $151.1 \pm 1.2$ & $154.1 \pm 1.8$ & $148.0 \pm 1.5$ & $<0.05$ \\
\hline Body weight (kg) & $43.5 \pm 1.2$ & $45.7 \pm 1.8$ & $41.3 \pm 1.5$ & 0.07 \\
\hline BMI $\left(\mathrm{kg} / \mathrm{m}^{2}\right)$ & $18.7 \pm 0.3$ & $18.9 \pm 0.5$ & $18.5 \pm 0.4$ & 0.50 \\
\hline Total IgE (IU/mL) & $407 \pm 64$ & $363 \pm 73$ & $452 \pm 105$ & 0.71 \\
\hline Allergic disease & & & & - \\
\hline BA & 6 & 5 & 1 & \\
\hline $\mathrm{AD}$ & 8 & 6 & 2 & \\
\hline $\mathrm{AR}$ & 35 & 17 & 18 & \\
\hline FA & 4 & 4 & 0 & \\
\hline Overall & 40 & 29 & 11 & \\
\hline None & 60 & 28 & 32 & \\
\hline
\end{tabular}

All values are presented as means \pm standard error (SE) with $P$ values calculated using $t$-test.

$\mathrm{AD}$, atopic dermatitis; $\mathrm{AR}$, allergic rhinitis; $\mathrm{BA}$, bronchial asthma; FA, food allergy.

The prevalence of current allergic disease was $6 \%$ for BA, $8 \%$ for $\mathrm{AD}, 35 \%$ for $\mathrm{AR}$, and $4 \%$ for food allergy (FA) (Figure 2). Of 6 early adolescents with BA, 5 (83.3\%) were male. Of 8 early adolescents with $\mathrm{AD}, 6(75.0 \%)$ were male. Of 35 early adolescents with AR, 17 (48.6\%) were males (Table 1). Forty early adolescents had one or more allergic diseases at the time of the study (Table 1). Of those 40 early adolescents, $29(72.5 \%)$ were male. The estimated lifetime prevalence was $27 \%$ for $\mathrm{BA}, 17 \%$ for $\mathrm{AD}, 46 \%$ for $\mathrm{AR}$, and $19 \%$ for $\mathrm{FA}$. There were $21,9,11$, and 15 early adolescents in remission for BA, $\mathrm{AD}, \mathrm{AR}$, and FA, respectively (Figure 2). Of these, 16 (76.2\%), 7 (77.8\%), $4(36.4 \%)$ and $9(60 \%)$ were male, respectively.

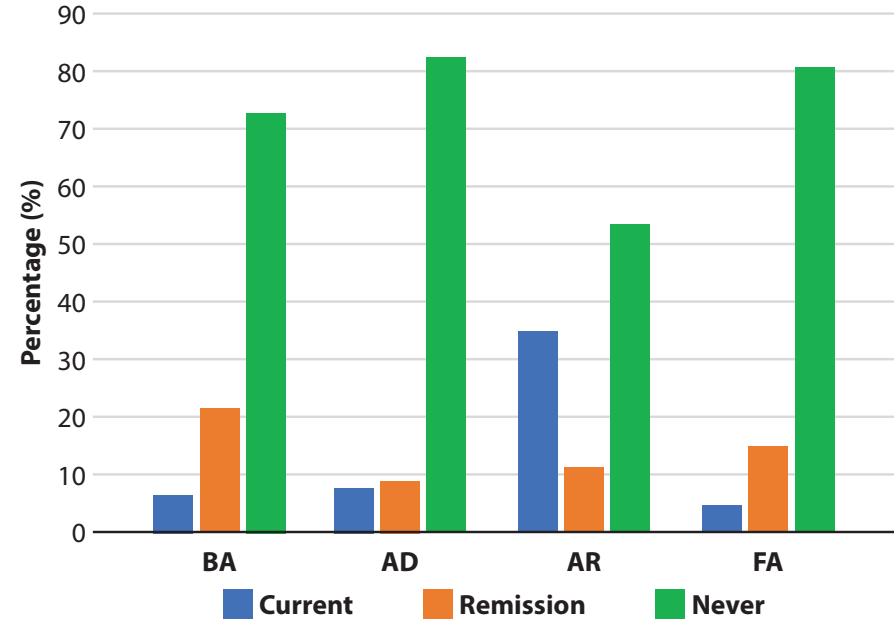

Figure 2. Current and lifetime prevalence of allergic diseases.

Current indicates currently having the condition. Remission indicates a previously diagnosed but currently asymptomatic condition. Never indicates having never had the condition. $\mathrm{AD}$, atopic dermatitis; $\mathrm{AR}$, allergic rhinitis; $\mathrm{BA}$, bronchial asthma; $\mathrm{FA}$, food allergy.

We investigated sensitization in participants with allergic diseases. The sensitization rate was the highest for Japanese cedar (51\%), followed by Dermatophagoides farinae, house dust mite, orchard grass, timothy grass, and cat. The sensitization rate for one or more antigens was $100 \%, 100 \%$, and $94.3 \%$ in participants with $\mathrm{BA}, \mathrm{AD}$, and $\mathrm{AR}$, respectively. There were 16 children who had no allergic symptoms and were not sensitized at all (Table 2). Of the 16 controls, 5 $(31.3 \%)$ were male.

Table 2. Serum periostin levels in early adolescents by allergic disease.

\begin{tabular}{lcc} 
& $\begin{array}{c}\mathbf{N} \\
(\%)\end{array}$ & $\begin{array}{c}\text { Serum periostin } \\
(\mathbf{n g} / \mathbf{m L})\end{array}$ \\
\hline $\begin{array}{l}\text { With one or more allergic disease } \\
\text { With one or more allergic diseases } \\
\text { (remission) }\end{array}$ & 40 & ${ }^{\star} 41.6 \pm 4.0$ \\
$\begin{array}{l}\text { With AR } \\
\text { Without allergic diseases }\end{array}$ & 39 & $* 41.4 \pm 4.5$ \\
$\begin{array}{l}\text { With no allergic disease and negative for } \\
\text { allergen-specific IgE }\end{array}$ & 60 & $32.7 \pm 2.6$ \\
\hline
\end{tabular}

Demographic data were presented as means \pm standard error (SE). ${ }^{\star} P<0.05$. Welch's t-test was used to compare the mean periostin levels in each group with the mean periostin level in the group with no allergic disease/sensitization*.

Remission indicates a previously diagnosed but currently asymptomatic condition.

AR, allergic rhinitis. 


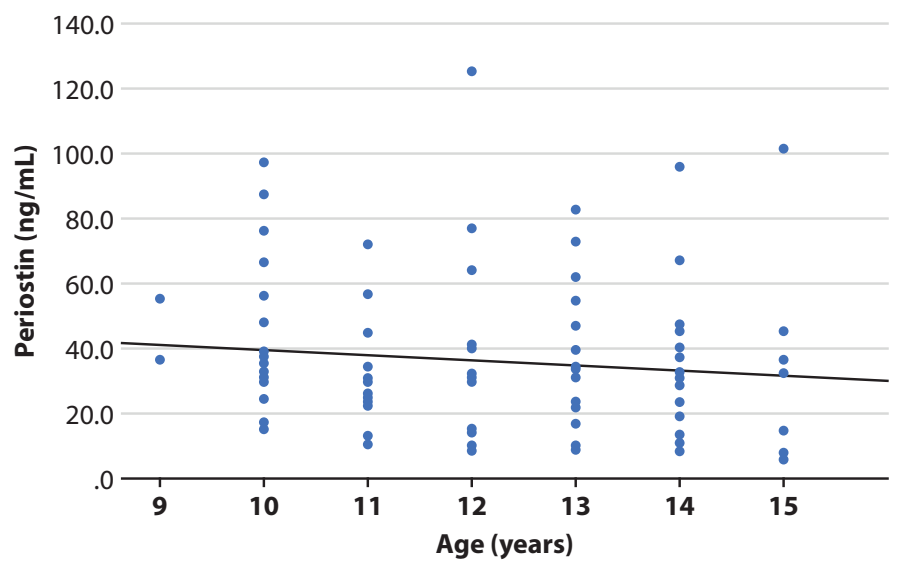

Figure 3. Association between serum periostin levels and age.

The mean serum periostin level was $36.2 \pm 2.3 \mathrm{ng} / \mathrm{mL}$. The average for males was $36.4 \pm 3.3 \mathrm{ng} / \mathrm{mL}$ and the average for females was $36.1 \pm 3.2 \mathrm{ng} / \mathrm{mL}$. There were no significant differences between males and females. Spearman's correlation test revealed no association between serum periostin levels and age $(\mathrm{r}=-0.11, P=0.24)$ (Figure 3) or BMI $(\mathrm{r}=$ $-0.12, P=0.24)$. Multivariated linear regression analysis revealed that age and BMI were not significantly associated with serum periostin levels [coefficient (95\%CI): -1.085 (-4.040, $-1.870),-0.705(-2.325,0.915)$, respectively]. The mean periostin level in participants with allergic diseases overall were significantly higher than in participants with no allergic diseases and negative allergen-specific IgE assay results (Table 2). Mean periostin levels in participants with current $A R$ and in remission for allergic disease was significantly higher than in participants that did not have allergic diseases/sensitization (Table 2).

$$
50
$$

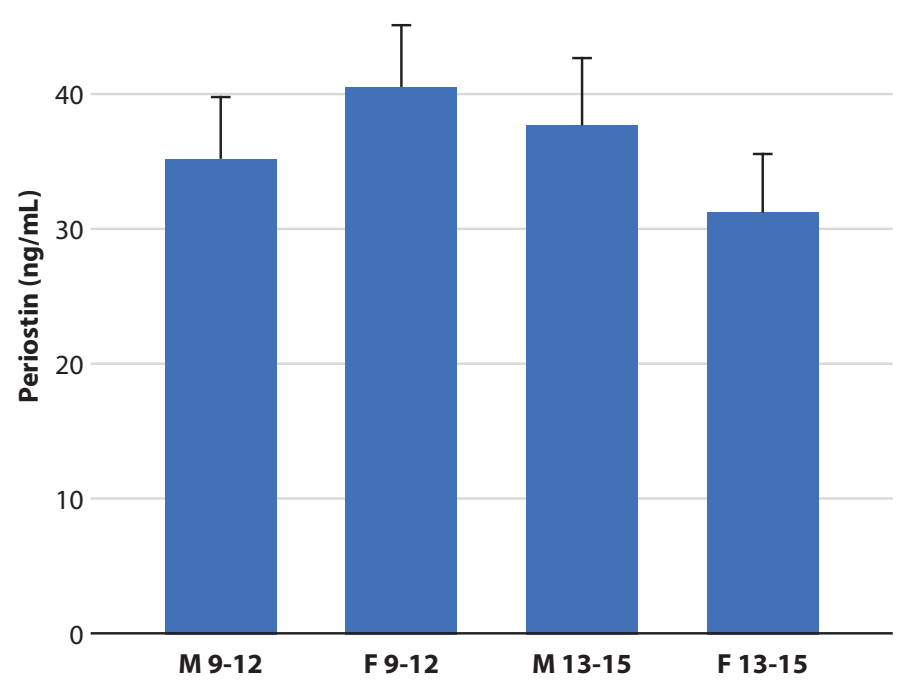

Figure 4. Serum periostin levels by age and gender groups. Error bars indicate standard error.
Using age 13 as the cutoff, there were 27 males and $26 \mathrm{fe}$ males in pre-early adolescence and 23 males and 24 females in post-early adolescence. The mean total IgE levels was 450 $\pm 92 \mathrm{IU} / \mathrm{mL}$ for those in pre-early adolescence and $360 \pm 88$ $\mathrm{IU} / \mathrm{mL}$ for those in post-early adolescence $(P=0.49)$. The mean periostin level for males and females in pre-early adolescence was $35.2 \pm 4.5 \mathrm{ng} / \mathrm{mL}$ and $40.5 \pm 4.6 \mathrm{ng} / \mathrm{mL}$ and $37.8 \pm 4.9 \mathrm{ng} / \mathrm{mL}$ and $31.4 \pm 4.1 \mathrm{ng} / \mathrm{mL}$ for males and females in post-early adolescence (Figure 4). Mean periostin levels were higher in females than males in pre-early adolescence and higher in males than females in post-early adolescence, but there were no significant differences among these four groups (Figure 4).

\section{Discussion}

Serum periostin levels were significantly elevated in participants in early adolescence who had one or more allergic diseases compared to those with no allergic diseases and negative allergen-specific IgE assay results. In this age group, periostin levels did not differ by age or gender.

Serum periostin levels were significantly higher in participants with allergic diseases as compared with healthy individuals in early adolescence. In previous reports, serum periostin levels were higher in patients with $\mathrm{AD}^{9}$ or $\mathrm{BA},{ }^{8,12}$ but not AR. ${ }^{13,14}$ In these surveys, ${ }^{8,9,13,14}$ allergic disease status was only based on questionnaire responses. Skin test and blood test were performed to confirm house dust mite sensitization in some studies ${ }^{8,12}$ related to asthma. The MAST immunosystems IV helped our diagnosis because our study included patients with AR and we did not limit participants' dose when they participated in this study. Many studies on the prevalence of allergic disease in various areas of Japan have used ISAAC criteria, such as in Tochigi $^{15}$ in 1995, Tokyo ${ }^{6}$ in 2005, and Ogasawara $^{16}$ in 2012. In this study, the prevalence of BA and $\mathrm{AD}$ was lower than in other regions, but the prevalence of AR was similar. In terms of gender, patients with BA and $\mathrm{AD}$ were more predominantly male, consistent with an earlier report. ${ }^{16}$ There were no differences in the prevalence of AR by gender, which was consistent with other reports. ${ }^{6,15}$ The geographic area of this study can be categorized as suburban to rural. The mean periostin level in this study was considerably lower than $134 \mathrm{ng} / \mathrm{mL}$, which was reported. ${ }^{8}$ One reason why serum periostin levels were lower in the group with allergic diseases is that this study included a large number of healthy individuals in early adolescence because it was an epidemiological survey in this age group. Other reasons include different methods of measuring serum periostin levels and different age ranges across studies.

There are no other reports of increased periostin levels in individuals in early adolescence with AR. However, Ishida et al. showed that periostin levels are significantly higher in tissues derived from patients with AR with nasal polyps than in control tissues. ${ }^{17}$ Moreover, periostin is likely to move easily from affected lesions into blood vessels. ${ }^{18}$ As a result, $\mathrm{AR}$ seems to be associated with increased periostin levels, reflecting a disease state. 
Serum periostin levels in patients with remission of allergic disease have not previously been reported. In bronchial biopsy samples from patients with asthma, the remission group have higher levels of periostin deposition than the healthy control group. ${ }^{19}$ We used an improved questionnaire to determine remission. We found that periostin levels are significantly elevated in individuals in early adolescence with allergic disease remission compared with healthy counterparts. The reason for the significant increase in remission is unknown. The short duration of remission of allergic symptoms might have affected periostin levels. Future research might be important and clarify intended meaning of as remission status.

Serum periostin levels were higher in early adolescents with allergic diseases than those with no allergic diseases and negative allergen-specific IgE assay results. Periostin is a matricellular protein that has been found in bone, connective tissue, myocardium, and skin. ${ }^{20,21}$ It has been shown to be highly expressed in BA. ${ }^{22}$ Since periostin is a molecule downstream of IL-4 and IL-13 and is involved in the pathogenesis of fibrosis, it has been established as a serum biomarker of remodeling in various allergic diseases in adults. ${ }^{3}$ However, it was pointed out that in children, an elevation due to allergic inflammation might be masked. ${ }^{14}$ Since periostin is produced by osteocytes and periosteal osteoblasts, ${ }^{23}$ serum periostin levels may be largely influenced by the rate of bone metabolism during adolescence. Peak height velocity (PHV) is an indicator of growth. It was reported the mean age of males at PHV is 11.8 years and that of females is 9.6 years in Japan. ${ }^{24}$ The age at which bone maturity peaks as indicated by the speed of sound in bone measured by ultrasound is supposedly 1 year after age at PHV. ${ }^{25}$ Serum periostin levels are expected to be elevated during age 9-12 years, but there were no significant differences observed in this study. Serum periostin levels have been reported to be higher in healthy infants. ${ }^{26}$ A study involving participants aged 16-18 years showed they have higher periostin levels than adults, suggesting the involvement of bone metabolism by Insulin-like Growth Factor-1. ${ }^{27}$ No studies have included all age groups throughout adolescence. Although, it was estimated that this age group had a high bone metabolism, serum periostin levels were used to distinguish allergic conditions.

Another feature of this study is the difference in how controls were defined. Previous reports differed in how controls were defined: by questionnaire, ${ }^{13,14}$ pediatrician judgment, ${ }^{9}$ or absence of other allergic diseases. ${ }^{8,26}$ These approaches may not adequately rule out the effects of sensitization. One reason is that periostin has been reported to be implicated in atopic conditions, such as rhinitis. ${ }^{17}$ We confirmed that there was no sensitization in healthy subjects in early adolescence without allergic diseases using MAST IV. Sensitization has been reported not to have an effect on periostin levels in AR..$^{13}$ In that report, 26 items were examined using a skin test. We evaluated sensitization in healthy adolescents with more rigorous blood test measurements in this study. We could confirm sensitization of more antigens by only one blood collection, without the need for multiple punctures. In this pediatric study, we were able to reduce the level of invasiveness. As a result, there were no significant differencesin mean periostin levels when participants in pre-early and post-early adolescence were compared. It was thought that periostin levels increased with allergic diseases. In early adolescents, basal periostin levels have been reported to be lowest at ages 7-10 and gradually increase after age $10 .^{3}$ Periostin levels differed with and without allergic disease, indicating that periostin alterrations were not masked in these age groups.

There are some limitations to this study. First, it was cross-sectional and observational, so causal relationships between serum periostin levels and allergic diseases cannot be inferred. Second, it was a single-center study. Results might not necessarily be generalizable to other regions. Further studies are needed to determine the reference value for periostin in early adolescents.

In conclusion, we found that serum periostin levels are significantly higher in early adolescents with allergic diseases. Serum periostin appears to be a biomarker that reflects allergic inflammation even at 9-15 years of age. The effects of bone metabolism on serum periostin levels may be limited in that age range.

\section{Acknowledgements}

We thank Hitachi Kasei for supporting the cost of measuring specific IgE antibodies. We are grateful to Dr. Enomoto for helpful advice on epidemiological investigations.

\section{Conflict of interest}

The authors have no conflict of interest to declare.

\section{Author contributions}

Yosuke Nakamura and Hiromi Takeuchi designed the study. Yosuke Nakamura wrote the manuscript. Yuko Yokoyama and Kenichiro Nakajima contributed to data collection. Yosuke Nakamura, Tadao Enomoto and Kazunori Fujiwara performed the statistical analysis and interpretation of the results. All authors read and approved the final manuscript.

\section{References}

1. Asher MI, Keil U, Anderson HR, Beasley R, Crane J, Martinez F, et al. International Study of Asthma and Allergies in Childhood (ISAAC): rationale and methods. Eur Respir J. 1995;8(3):483-91.

2. Izuhara K, Arima K, Ohta S, Suzuki S, Inamitsu M, Yamamoto K-i. Periostin in Allergic Inflammation. Allergol Int. 2014;63(2):143-51.

3. Fujitani H, Kasuga S, Ishihara T, Higa Y, Fujikawa S, Ohta N, et al. Age-related changes in serum periostin level in allergic and non-allergic children. Allergol Int. 2019;68(2):285-6

4. Beasley R. Worldwide variation in prevalence of symptoms of asthma, allergic rhinoconjunctivitis, and atopic eczema: ISAAC. The Lancet. 1998;351(9111):1225-32.

5. Kusunoki T, Morimoto T, Nishikomori R, Yasumi T, Heike T, Fujii T, et al. Changing prevalence and severity of childhood allergic diseases in kyoto, Japan, from 1996 to 2006. Allergol Int. 2009;58(4):543-8.

6. Futamura M, Ohya Y, Akashi M, Adachi Y, Odajima H, Akiyama K, et al. Age-related prevalence of allergic diseases in Tokyo schoolchildren. Allergol Int. 2011;60(4):509-15.

7. Lopez-Guisa JM, Powers C, File D, Cochrane E, Jimenez N, Debley JS Airway epithelial cells from asthmatic children differentially express proremodeling factors. J Allergy Clin Immunol. 2012;129(4):990-7 e6.

8. Inoue T, Akashi K, Watanabe M, Ikeda Y, Ashizuka S, Motoki T, et al. Periostin as a biomarker for the diagnosis of pediatric asthma. Pediatr Allergy Immunol. 2016;27(5):521-6. 
9. Sung M, Lee KS, Ha EG, Lee SJ, Kim MA, Lee SW, et al. An association of periostin levels with the severity and chronicity of atopic dermatitis in children. Pediatr Allergy Immunol. 2017;28(6):543-50.

10. Anderson HM, Lemanske RF Jr, Arron JR, Holweg CTJ, Rajamanickam V, Gangnon RE, et al. Relationships among aeroallergen sensitization, peripheral blood eosinophils, and periostin in pediatric asthma development. J Allergy Clin Immunol. 2017;139(3):790-6.

11. Okubo K, Kurono Y, Ichimura K, Enomoto T, Okamoto Y, Kawauchi $\mathrm{H}$, et al. Japanese guidelines for allergic rhinitis 2017. Allergol Int. 2017; 66(2):205-19.

12. Song JS, You JS, Jeong SI, Yang S, Hwang IT, Im YG, et al. Serum periostin levels correlate with airway hyper-responsiveness to methacholine and mannitol in children with asthma. Allergy. 2015;70(6): 674-81.

13. Kim DY, Kim JH, Lee KH, Hong SC, Lee HS, Kang JW. Serum periostin level is not associated with allergic rhinitis or allergic sensitization in Korean children. Int J Pediatr Otorhinolaryngol. 2017;93:24-9.

14. Inoue $\mathrm{Y}$, Izuhara $\mathrm{K}$, Ohta S, Ono J, Shimojo N. No increase in the serum periostin level is detected in elementary school-age children with allergic diseases. Allergol Int. 2015;64(3):289-90.

15. Sugiyama K, Sugiyama T, Toda M, Yukawa T, Makino S, Fukuda T. Prevalence of asthma, rhinitis and eczema among 13-14-year-old schoolchildren in Tochigi, Japan. Allergol Int. 2000;49(3):205-11.

16. Okada Y, Kumagai H, Morikawa Y, Akasawa A. Epidemiology of pediatric allergic diseases in the Ogasawara Islands. Allergol Int. 2016; 65(1):37-43.

17. Ishida A, Ohta N, Suzuki Y, Kakehata S, Okubo K, Ikeda H, et al Expression of pendrin and periostin in allergic rhinitis and chronic rhinosinusitis. Allergol Int. 2012;61(4):589-95.
18. Izuhara K, Matsumoto H, Ohta S, Ono J, Arima K, Ogawa M. Recent developments regarding periostin in bronchial asthma. Allergol Int. 2015; 64 Suppl:S3-10.

19. Evans S. Periostin as a marker of remodeling in young adults with clinical remission of asthma. 2013;42(Suppl 57):P584.

20. Kii I, Amizuka N, Minqi L, Kitajima S, Saga Y, Kudo A. Periostin is an extracellular matrix protein required for eruption of incisors in mice. Biochem Biophys Res Commun. 2006;342(3):766-72.

21. Kikuchi Y, Kashima TG, Nishiyama T, Shimazu K, Morishita Y Shimazaki $\mathrm{M}$, et al. Periostin is expressed in pericryptal fibroblasts and cancer-associated fibroblasts in the colon. J Histochem Cytochem. 2008;56(8):753-64.

22. Takayama G, Arima K, Kanaji T, Toda S, Tanaka H, Shoji S, et al Periostin: a novel component of subepithelial fibrosis of bronchial asthma downstream of IL-4 and IL-13 signals. J Allergy Clin Immunol. 2006;118(1):98-104.

23. Merle B, Garnero P. The multiple facets of periostin in bone metabolism. Osteoporos Int. 2012;23(4):1199-212.

24. Yokoya M, Higuchi Y. Geographical Differences in the Population-Based Cross-Sectional Growth Curve and Age at Peak Height Velocity with respect to the Prevalence Rate of Overweight in Japanese Children. Int J Pediatr. 2014;2014:867890.

25. Kaga $M$, Kamimura $H$. Bone mass in puberty. Clin Calcium. 2008;18(8):1193-9.

26. O'Connell P, Gaston B, Bonfield T, Grabski T, Fletcher D, Shein SL. Periostin levels in children without respiratory disease. Pediatr Pulmonol. 2019;54(2):200-4.

27. Walsh JS, Gossiel F, Scott JR, Paggiosi MA, Eastell R. Effect of age and gender on serum periostin: Relationship to cortical measures, bone turnover and hormones. Bone. 2017;99:8-13. 